\title{
Precise Fluid-Solid Simulation of Human Left Ventricle along with Aortic Valve during Systole
}

\author{
$02+\$ 00$ \$ ' \$ , [0 21 ) \$ 5 ( ' A, MOHAMMAD MEHDI ALISHAHI A,1, \\ MARZIEH ALISHAHI ${ }^{\text {A }}$ \\ a School of Mechanical Engineering, \\ Shiraz University, \\ Shiraz, 71936-16548, IRAN
}

\begin{abstract}
This paper presents an accurate blood flow model with tissue deformation of the human left ventricle, including the aortic valve. A two-way fluid-solid Interaction (FSI) algorithm is employed to simulate the performance of the human left ventricle during systole. The initial geometry of the left ventricle is extracted from CT scan images of a healthy person. The simulation results produced the systolic anterior motion of the Left Ventricle (LV) identical with the CT scan images at later times during systole. Besides, the numerical results for left ventricular volume change, maximum blood velocity at the aortic valve, and its maximum opening are in good agreement with physiological data. Although no clear image of the aortic valve is apparent in CT images, the FSI simulation predicted the maximum opening of the aortic valve to be $4.38 \mathrm{~cm}^{2}$ which is consistent with physiological observation on a healthy individual. As an application of the above algorithm, a model of Hypertrophic Cardiomyopathy (HCM) or septal wall thickening disease is constructed and studied during systole. This simulation provides an understanding of heart performance under HCM conditions. According to the simulation outcomes, the mitral valve approaches the septal wall under HCM due to the change in pressure gradient and the drag force on the mitral valve. This blockage of the LV blood passage by the mitral valve results in stagnation pressure loss and weaker hearth pumping power. Therefore, the maximum opening of the aortic valve, in this case, is $2.28 \mathrm{~cm}^{2}$, which is much lower than the physiological range, indicating the drastic effect of HCM on the performance of the aortic valve and systolic performance.
\end{abstract}

Keywords: CFD, Systole, Left ventricle, Aortic valve, Fluid-Solid interaction, Mitral valve.

Received: April 15, 2021. Revised: January 12, 2022. Accepted: January 22, 2022. Published: March 3, 2022. 


\section{Introduction}

Cardiovascular diseases are the most crucial cause of death, about 17.9 million people, an estimated $31 \%$ of all deaths worldwide. Therefore, any attempt to provide a better knowledge of how they affect the regular operation of the heart is worthwhile. One of the disciplines for such studies is the biomechanical branch and specifically the heart simulation. In the end, such the ability to predict the malfunction of the diseased heart would be invaluable for choosing a cure strategy.

With the ongoing growth of computational capacities, researchers are inclined to use Computational Fluid Dynamics (CFD) methods and especially Fluid-Solid Interaction (FSI) to simulate blood flow in different parts of the cardiovascular system [1-3]. These methods are also employed to investigate heart pumping function in a simplified model of the Left Ventricle (LV) along with the aortic valve using different geometrical assumptions [4]. Watanabe et al. [5] assumed a simplified geometry model and investigated the ventricular volume change due to different contractile-stimulus using the FSI method. The computational algorithm was based on the EulerianLagrangian finite element method, while the dynamic meshing captured large deformations. Moosavi et al. [6] constructed an anatomical model of the $\mathrm{LV}$ and the aortic sinus based on the real images of a volunteer and carried out the numerical simulations of blood flow in the model. The computed results for aortic outflow were compared with the data obtained by the phase-contrast MRI, and an acceptable agreement between the simulation results and the physiological measurements was reported. Lorenz et al. [7] presented a cardiac model consisting of four cardiac chambers, cardiovascular and coronary arteries. This geometric heart model was built based on data from $27 \mathrm{CT}$ cores at the end of the diastole. The model's accuracy was measured based on the comparison with different references.

There is also some research on more precise aortic valve modeling. Kim et al. [8] simulated the nonlinear structure of polymer aortic valves with three leaflets using both computational and experimental approaches. They presented a structural model that can predict the heart structure's transformation with a maximum error of $10 \%$ during one complete stage of heart contraction. Moreover, another research carried out by Merryman et al. [9] studied the function of the heart valves by estimating at least $3 \times$ $10^{9}$ cycles for one person's life. Focused on the biocompatibility of heart valves, i.e., bio-solid along with the bio-fluid characteristics, the physiological function of the aortic valve was well-documented. Trung Bao Le et al. [10] investigated a realshaped LV with a simple aortic valve model, two sheets that open and close, using the FSI method. In this modeling, the mitral valve was assumed to be completely open during diastole and completely closed during systole. The results showed an asymmetry in the closure of aortic valve leaflets, which did not match the physiological results.

Considering the material models available for valve's leaflet tissue, Sturla et al. [11] assumed the mechanical response of the aortic valve to be linear, and therefore elastic material was an appropriate model. 
Employing the FSI method, it was shown that the use of elastic material for valve leaflets gives relatively good results compared to experimental results. MC Hsu et al. [12] developed a hyperelastic model of a biological aortic valve placed at the opening of the aorta. Two material models were chosen for aortic valve tissue; Fung and Venant-Kirchhoff models. It was observed that the Venant-Kirchhoff model produced relevant results. Using the FSI method with the Venant-Kirchhoff model for the aortic valve, the outcomes were remarkably close to the measured in vivo data.

The main objective of the current study is to present and investigate a real-shaped model of $\mathrm{LV}$ along with an aortic valve during systole. The initial 3-dimensional geometry is constructed based on CT scan images of a healthy person to fulfill this purpose. Blood is considered a Newtonian fluid, and the hyperelastic Ogden model is chosen for ventricular wall tissue and aortic valve leaflets. The blood flow inside the LV and ventricular wall tissue movement were simulated using a two-way FSI algorithm, and the obtained results are compared with physiological data as a verification of the modeling and the simulation. Thorough apprehension of aortic valve plus LV operation provide a more precise tool for studying different diseases and could assist heart valve pre-surgery evaluations. Along this path, a model of septal wall thickening disease, hypertrophic cardiomyopathy (HCM), is also constructed and investigated during the systole. HCM is a heart muscle disease where the myocardium becomes thickened, resulting in stiffer heart muscle. If HCM occurs in the $\mathrm{LV}$, it also will reduce the ventricular volume up to $50 \%$ [13]. In the case of HCM, the mitral valve leaflets approach the septum wall and partially block the blood flow into the aorta, and eventually, the heart will lose its pumping ability. There are two potential causes for such phenomena; the change in pressure gradient and drag force hypothesis [14] resulting in total pressure loss in systolic performance. In this paper, a simple form of HCM in LV is modeled at the start of the systole, and the resulting dislocation of mitral valve leaflets and heart performance degradation is investigated.

\section{Physical Modeling}

\section{2-1 Left Ventricle Geometry}

In this study, the geometry of the left ventricle is extracted from CT scan images of a healthy young male at the beginning of systole. The CT scan images are taken by a Philips Brilliance 64-slices CT scanner. The images are captured in 21 phases, and each phase contains 397 slices in three different views. The first and last phases are identical. The corresponding time and spatial resolution are $38 \mathrm{~ms}$ and $0.42 \mathrm{~mm}$, respectively. The left ventricle geometry was constructed from a CT angiography set of images at the beginning of the systole phase, using Mimics software. Figure 1 shows the CT images taken from one volunteer at the beginning of the systole. The red color represents the blood flow region, and the violet color identifies the left ventricle wall tissues. 
(a)

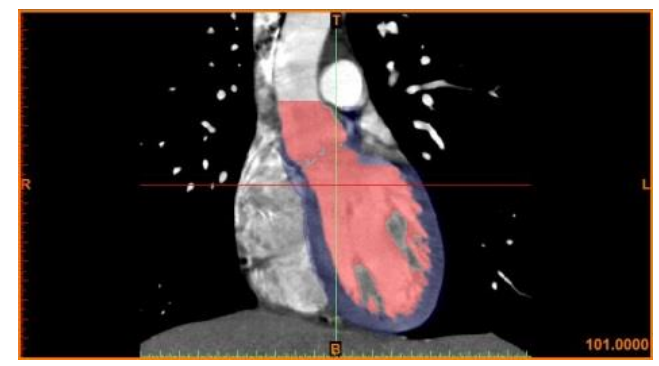

(c)

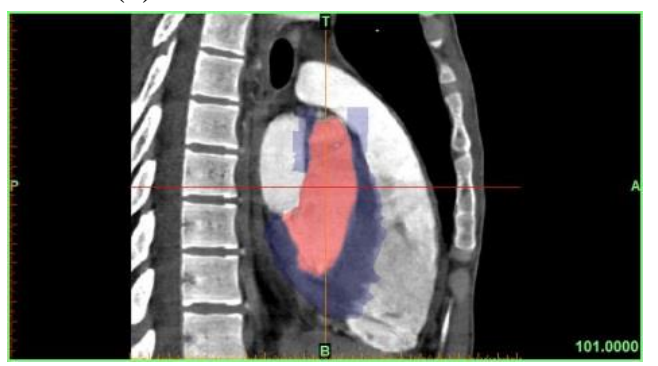

(b)

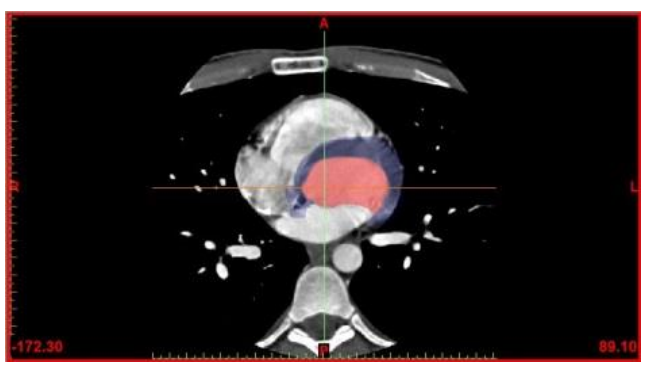

(d)

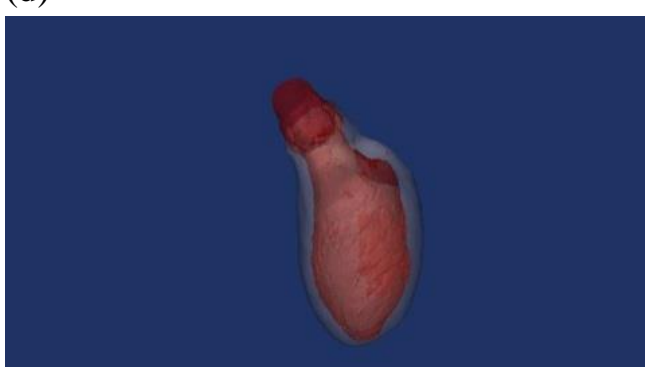

Figure 1 Different views of left ventricle (a) Front view (b) Top view (c) Side view (d) 3D view.
The smoothed left ventricle geometries and the ventricular volume, which is the blood

(a)

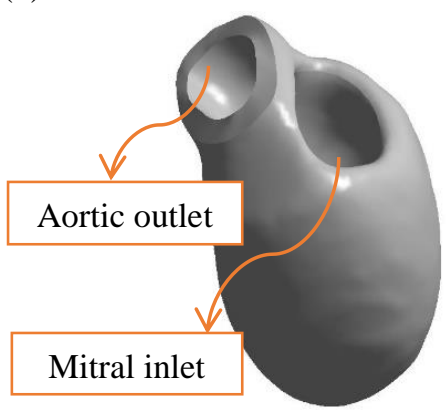

region at the beginning of systole, are shown in figure 2.

Figure 2 Left ventricle geometry (a) solid region (b) fluid region.

The HCM model is constructed based on the geometry of reference [14] and is shown in figure 3. It is noteworthy that the flexible (b)

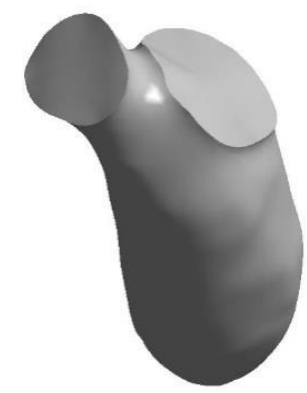

mitral valve is modeled for HCM study in order to examine the septum and mitral valve interactions. 
(a)

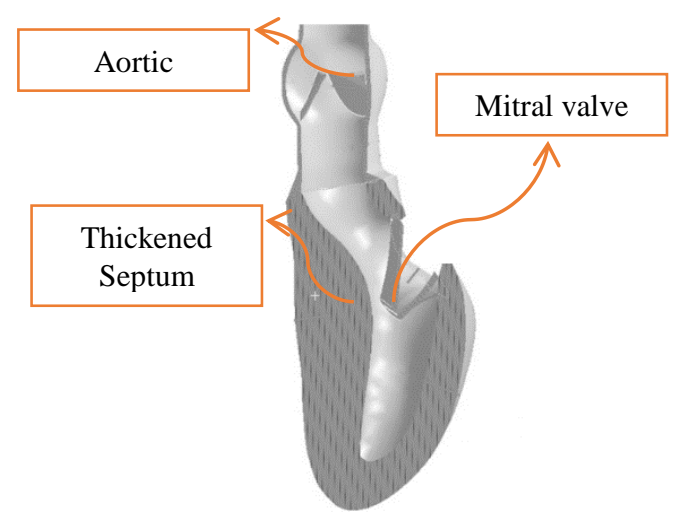

(b)

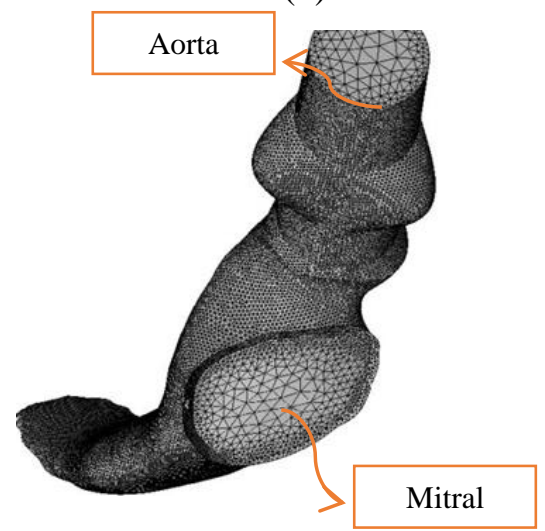

Figure 3 Left ventricle geometry for HCM model (a) solid region (b) fluid region.

\section{2-2 Aortic Valve Geometry}

The aortic valve has a complex structure, but due to the rapid opening and closure, proper medical images of the valve could not be extracted from CT images. Therefore, aortic valve geometry is constructed with the aid

(a)

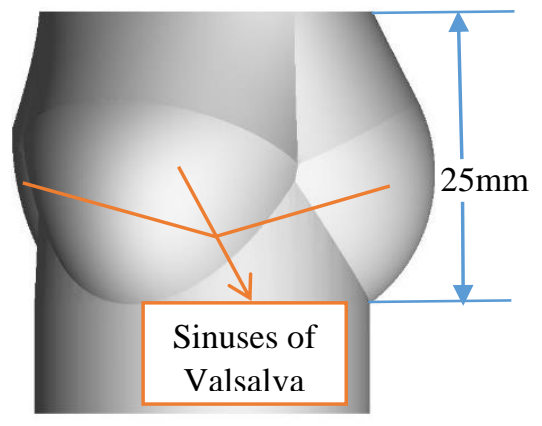

(b)

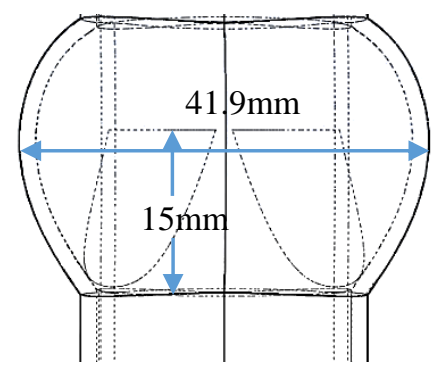

of CAD software, enclosed by boundaries of CT scan images, and based on physiological information. The dimensions of different parts of the reconstructed aortic valve are shown in figure 4.

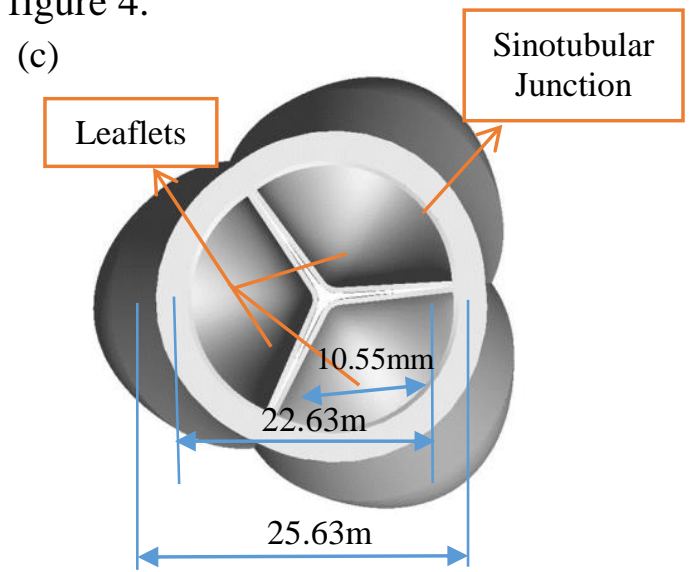

Figure 4 Aortic valve model (a) and (b) front view (c) top view.

After reconstruction of the aortic valve, it was integrated with the left ventricles in
CAD software. The left ventricular geometry and added aortic valve are shown in figure
5. 
(a)

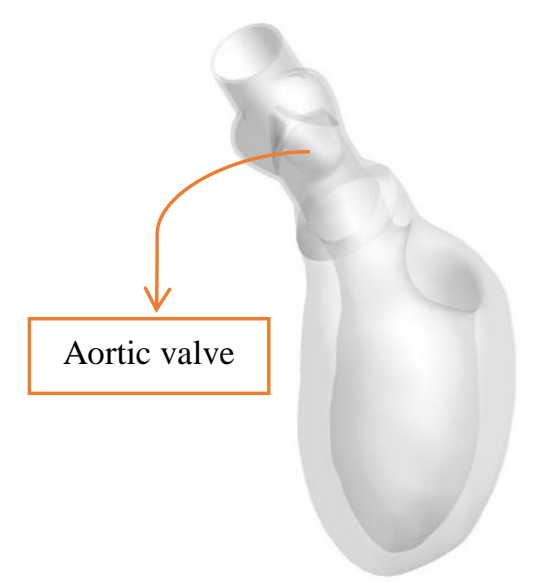

(b)

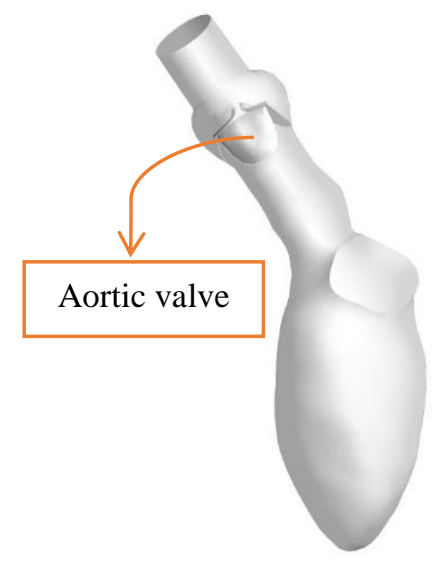

Figure 5 Left ventricle geometries with the aortic valve (a) solid domain (b) fluid domain.

\section{2-3 Material Models}

Blood is modeled as an isothermal, incompressible and Newtonian viscous fluid with density of $1060 \mathrm{Kg} / \mathrm{m}^{3}$ and a dynamic viscosity of 0.00345 Pa.s [15-16]. Blood flow is assumed to be laminar $[6,17]$.

During systole and diastole, the heart muscles undergo large deformations; therefore, the hyperelastic material model is the best choice to simulate these behaviors. For the sake of simplicity, the material of the aortic valve and left ventricular wall is assumed to be homogenous, isotropic, and hyperelastic. This study uses the Ogden model for the hyperelastic strain energy function for the aortic valve and left ventricular wall. According to Ogden model, the strain energy is defined as;

$W=\sum_{i=1}^{N} \frac{\mu_{i}}{\alpha_{i}}\left(\bar{\lambda}_{1}^{\alpha_{i}}+\bar{\lambda}_{1}^{\alpha_{i}}+\bar{\lambda}_{1}^{\alpha_{i}}-3\right)+\sum_{i=1}^{N} \frac{1}{d_{i}}(J-1)^{2 i}$

Where $\alpha_{i}$ and $\mu_{i}$ are time dependent variables, $d_{i}$ is the material compressibility parameter and $\lambda_{i}$ is principal stretch. The $\mu_{0}=\frac{1}{2} \sum_{i=1}^{N} \alpha_{i} \mu_{i}$ $K_{0}=\frac{2}{d_{1}}$

The corresponding parameters of the stressstrain relation were previously evaluated using the uniaxial experiment data [18-19] initial shear modulus, $\mu_{0}$ and initial bulk modulus $K_{0}$ are;

Table 1. Ogden model parameters.

\begin{tabular}{|c|c|c|c|}
\hline $3^{\text {rd }}$ Order Ogden model & $\mathrm{i}=1$ & $\mathrm{i}=2$ & $\mathrm{i}=3$ \\
\hline$d_{i}\left(\mathrm{~Pa}^{-1}\right)$ & $4.56 \mathrm{E}-06$ & $1.49 \mathrm{E}-06$ & $-2.814 \mathrm{E}-06$ \\
\hline$\alpha_{i}$ & 7.59 & 7.62 & 7.61 \\
\hline$\mu_{i}(\mathrm{~Pa})$ & 81.88 & 81.88 & 81.89 \\
\hline
\end{tabular}




\section{Numerical Modeling}

This study applies the iterative segregated method to solve the structure and fluid equations using ANSYS software separately. The coupling of fluid and solid domains is carried out based on the two-way FSI method [21]. Accordingly, the latest results computed from one part of the coupled system are transmitted to the other part, and these communications are $\nabla \cdot \vec{u}=0$

$\rho_{b} \frac{\partial \vec{u}}{\partial t}+\rho_{b} \cdot(\vec{u} . \nabla) \vec{u}=-\nabla \mathrm{p}+\mu \nabla^{2} \vec{u}$ Where $\vec{u}$ is the velocity vector, $\mathrm{p}$ is the pressure, $\rho_{b}$ and $\mu$ are the density and the viscosity of blood, respectively. The equations (1) and (2) are solved by a transient, pressure based, finite volume algorithm.

\section{3-2 Boundary Conditions (BC)}

During the systole of a healthy heart, the mitral valve is closed to prevent backflow to the atrium while the aortic valve is open. Therefore, the mitral valve is assumed as the wall boundary condition with no flow through in the present study. At the aortic

(a)

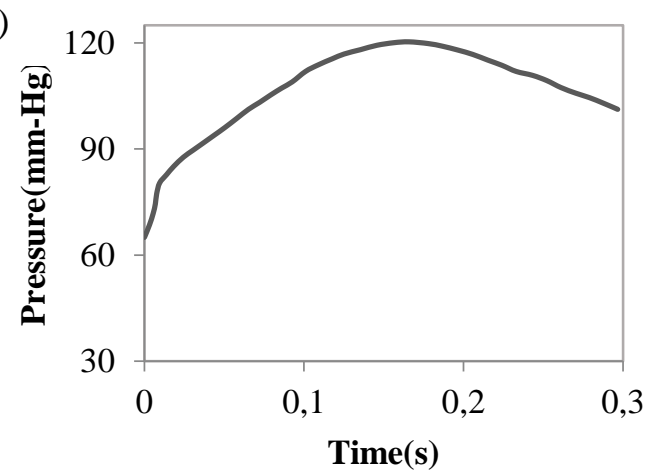

iteratively continued until the desired convergence criterion is reached. The whole simulation is carried out for a total physical time of $0.3 \mathrm{~s}$, covering the whole systolic period.

\section{3-1 Governing Equations}

The turbulent flow field of blood in LV is described by the 3D Navier-Stokes equations;

outlet, two different types of $\mathrm{BC}$ are used to compare and evaluate the simulation results with scan images. The first boundary condition is the velocity $\mathrm{BC}$, and the other is the pressure $\mathrm{BC}$ at the aortic valve. Two outlet BCs are the time-dependent physiological velocity and physiological pressure described respectively by Sagawa et al. [22] and Fielden et al. [23]. Other researchers widely used these relations for blood flow simulation inside the aorta. Figure 6 shows the physiological charts for average pressure and average velocity during systole.

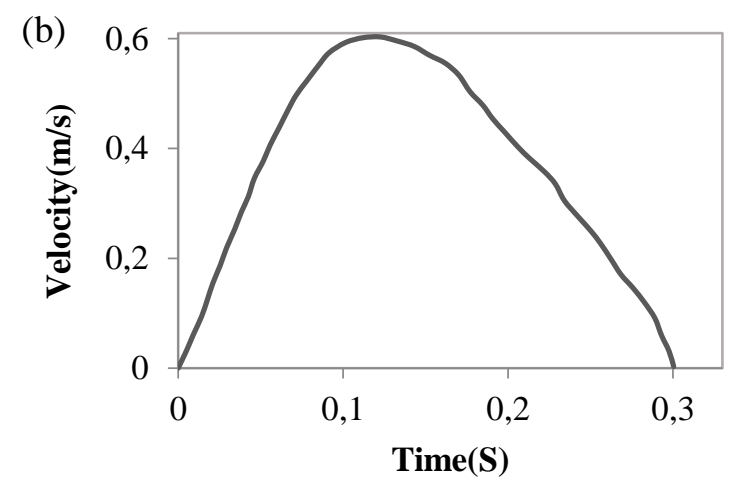

Figure 6 The physiological charts during systole; (a) Average pressure BC [22] (b) Average velocity BC [23]. 
The inner surfaces of the ventricular wall and the fluid surface in contact with this wall are assigned as fluid-structure interaction BC. The summary of the boundary conditions is demonstrated in figure 7 . The same BCs are defined for the HCM model.

(a)

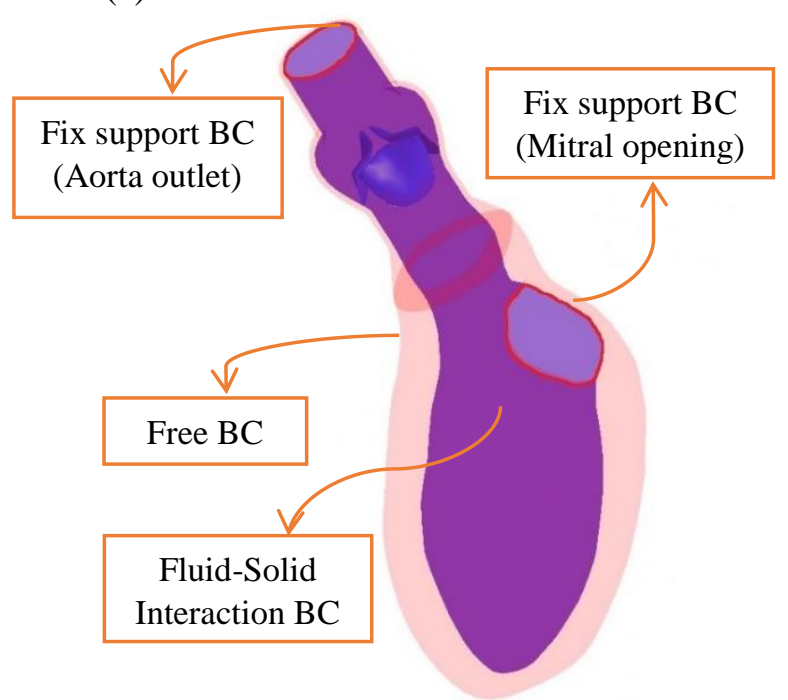

The FSI boundary condition can be described as;

$u_{f}=u_{s}$

$$
n \cdot \tau_{f}=n \cdot \tau_{s}
$$

In the above equations, $u_{f}$ and $u_{s}$ are fluid and solid displacements, and $\tau_{f}$ and $\tau_{s}$ are fluid and solid stress tensors, respectively.

(b)

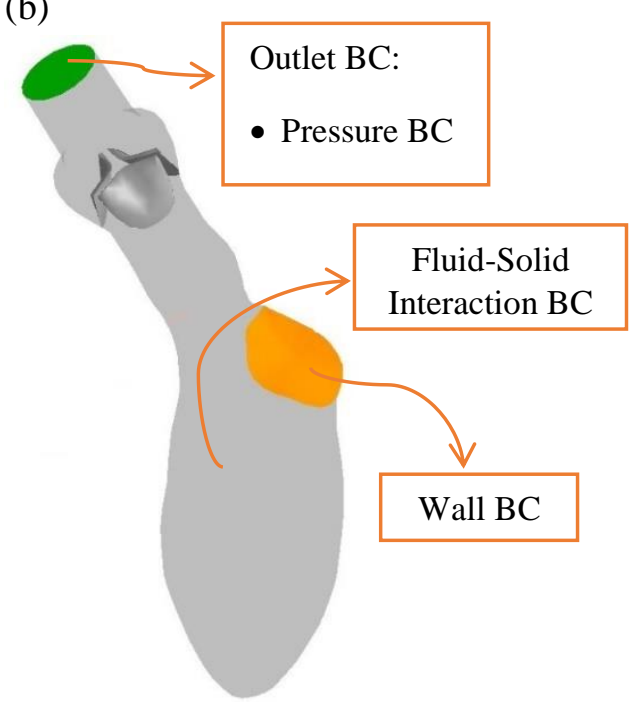

Figure 7 The boundary conditions; (a) solid boundary conditions (b) fluid boundary conditions

\section{3-3 Computational Grid}

For FSI modeling, the computational grids are generated for fluid and solid domains, and grid independence analysis was carried out separately. The unstructured grids are constructed for both solid and fluid regions. The fluid grid consists of prismatic and tetrahedral elements. The prismatic elements are built near the wall surface to capture the flow details in this region. The discretized equations are solved for each cell of the computational grid. Three different grids in both domains are generated to ensure that the obtained results are independent of the computational grid. Two surfaces are defined in the fluid domain, one near the apex and another near the aortic valve, to study the grid independence of fluid domain solution (figure 8). Figure 9 shows the grid independence results regarding areaweighted velocity computed on the described planes at time $0.12 \mathrm{~s}$. 
Plane 2

Figure 8 Position of planes used for grid independence analysis.

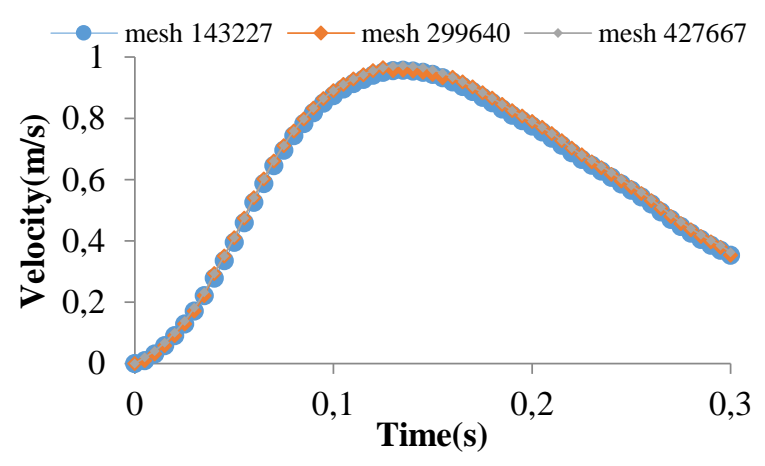

(a)

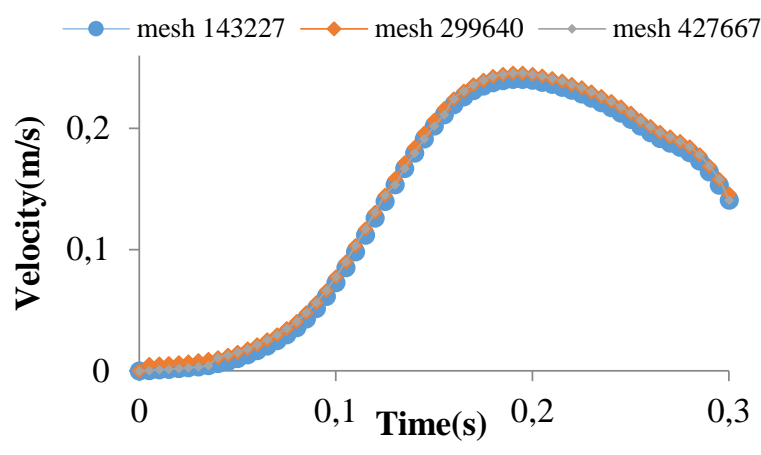

(b)

Figure 9 Mesh independency analysis based on average velocity changes on (a) plane 1 (b) plane 2.

Three grids with 143227, 299640, and 427667 elements are built to determine the optimal spatial mesh resolution. According to figure 9, the results of the three grids are identical, which means that the obtained solution is independent of the fluid grid. Also, in two grids with more elements, the results are closer to each other. The average difference in velocity magnitudes between the model with 299640 elements and the model with 427667 elements is less than 1\%. Therefore, the grid with 427667 elements is selected for further study. It should be noted that at the end of the systole, the speed on planes 1 and 2 is not zero, which is due to the incomplete aortic valve closure.

Grid independence analysis is also performed for the solid domain. Therefore, three different solid grids were generated with 107699, 151689, and 226413 elements. The results for the solid domain are presented in Table 2 for different numbers of elements for the LV wall tissue and aortic valve.

Table 2. Mesh study of the solid domain for the physical model.

\begin{tabular}{|c|c|c|c|}
\hline $\begin{array}{c}\text { Number of heart } \\
\text { muscle elements }\end{array}$ & $\begin{array}{c}\text { Number of aortic valve } \\
\text { elements }\end{array}$ & $\begin{array}{c}\text { Maximum heart muscle } \\
\text { deformation (mm) }\end{array}$ & $\begin{array}{c}\text { Maximum deformation } \\
\text { of aortic valve (mm) }\end{array}$ \\
\hline 66161 & 41583 & 20.612 & 5.88 \\
\hline 88264 & 63425 & 20.625 & 6.05 \\
\hline 111122 & 115291 & 20.628 & 6.08 \\
\hline
\end{tabular}


A similar grid independence analysis is also carried out for the HCM model. Three grids for the fluid domain are constructed; 80939, 190217, and 291154 elements. The results of the grid with 190217 elements are independent of grid size, and it is considered for further simulations.

Moreover, three time-steps, 0.0002 s, 0.0005 $\mathrm{s}$, and $0.001 \mathrm{~s}$, were chosen to study the time step independence of the simulation outcomes. It was observed that the simulation results with the time-step 0.0002 s were appropriate.

\section{Result and Discussions}

\section{4-1 Solid Domain}

The time variation of different parameters related to the aortic valve and myocardium tissue are shown in figure 10. At each node, the deformation is calculated in three Cartesian directions, and the maximum value of the resultant deformations in the entire geometry is reported as the maximum deformation at any given time (figure 10a

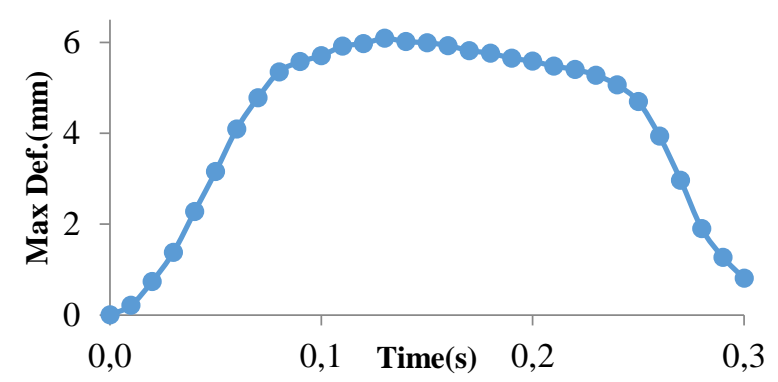

(a) and 10c). Based on figure 10, the deformation profile of the aortic valve is almost similar to the output velocity profile (figure 9a). The maximum deformation of the aortic valve occurred at $0.12 \mathrm{~s}$, at the same time where the output velocity is maximum (figure 9a). The following fact can explain it; as the aortic valve continues opening and its deformation increases to a maximum, the output velocity increases too and reaches a maximum without any time lag. However, the aortic valve opening happens in the numerical model due to the velocity or pressure $\mathrm{BC}$ at the outlet. It is noteworthy that the deformation of the aortic valve gradually decreases after this maximum (figure 10a) while the velocity drop is steeper (figure 9a) due to relaxation of the heart muscle that is clearly shown in figure $10 \mathrm{~d}$, after $\mathrm{t}=0.12 \mathrm{sec}$. Note that the early stage of systole, i.e., isovolumetric compression that happens quite quickly, is not precisely modeled here, although the steep pressure rises before $\mathrm{t}=9.5$ millisecond (figure 6a) somehow takes this effect into account.

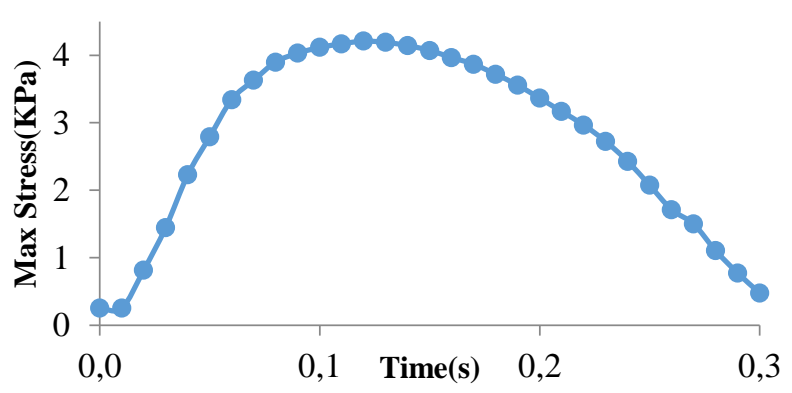

(b) 


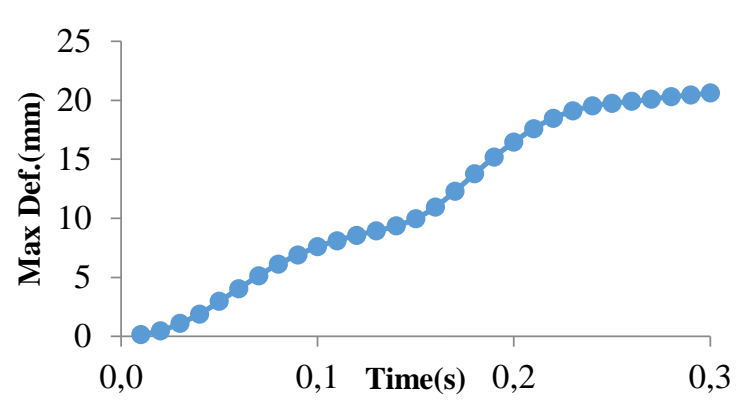

(c)

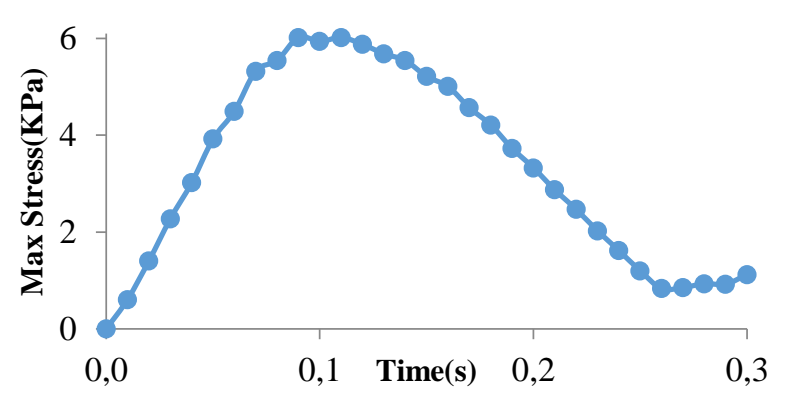

(d)

Figure 10 (a) Maximum deformation of the aortic valve (b) Maximum Tension in Aortic Valve Tissue (c) Maximum deformation of myocardium tissue (d) Maximum tension in myocardium.

According to figure 10a, the deformation is almost zero at the end of the systole in accordance with zero velocity at the aortic valve. Note that a closed valve position might accompany zero outflow. However, negative cell volume may occur in the grid in the event of complete closure of the aortic valve. The proper precautions are taken to avoid this erroneous situation in the current study. At the start of the systole period, this problem is handled by initializing the flow field with a negligible velocity.

The blood volume in the left ventricle for the specific person during systole was obtained by Mimics software. To valid the numerical outcomes, they should be compared with the measured data of the that individual whose CT scan images are used for this research. The for numerical simulations and measured data for blood volume changes in the left ventricle are shown in figure 11. According to figure 11, the ventricular volumes decrease monotonically in accordance with physiological predictions for both simulations with different BCs. This is in line with the increasing deformation of myocardium tissue shown in figure 10c and stress relaxation in figure 10d. Moreover, the trends of the simulation curves in figure 11 show a good agreement with the physiological data and the experimental data of the specific case.

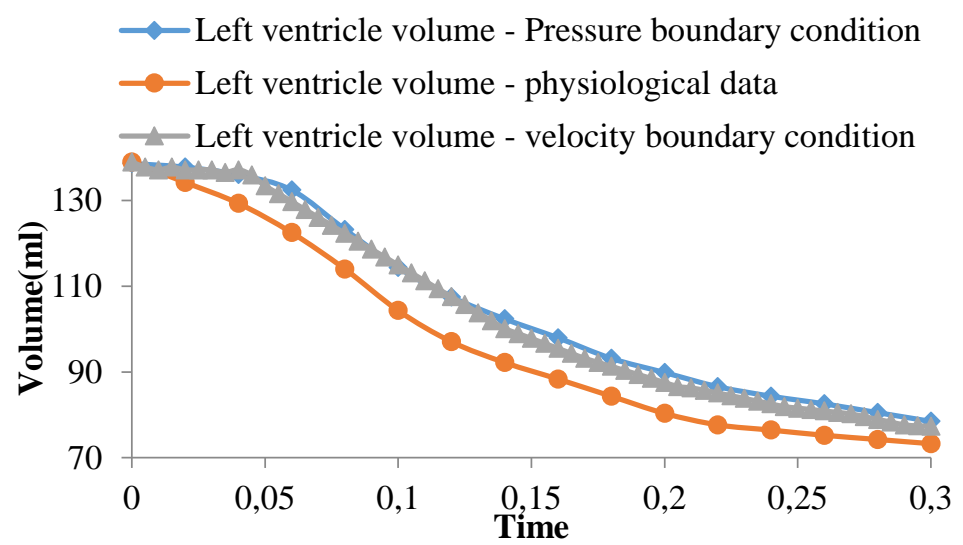

Figure 11 The outlet BC effect on the computed left ventricular volume change

Secondly, as shown in figure 11, there is a slight difference between the computed systolic volume of $\mathrm{LV}$ with velocity $\mathrm{BC}$ and pressure BC. Meanwhile, the difference of these two simulations with physiological 
data is more sensible, $10 \%$ maximum which tends to be smaller at the end of systole. However, the actual LV shape variation during systole is different for these two $\mathrm{BC}$ 's, explained later.

This is expectable since by defining a specific time variation of velocity at a constant sectional area such as the aorta, i.e., velocity $\mathrm{BC}$, we define the ventricle volume change; hence the relative agreement of velocity $\mathrm{BC}$ and the physiological predictions was seen. However, one expects more realistic results for pressure $\mathrm{BC}$ due to more freedom for flow field allowed by such BC.

Figure 12 demonstrates the aortic valve deformations during systole for simulations with applying pressure BC. The CT scan images from [24] are also added for comparison. The overall agreement is apparent.

In Figure 13, LV deformations during systole with outlet pressure BC are shown, which can be compared with the corresponding CT scan images collected in this study. Different views are provided in order to have a better comparison. Initial LV shapes in all views, at $\mathrm{t}=0$ Sec., are exactly the same as the CT images, and at later times differences between numerical and CT images show the simulation discrepancies. According to the CT scan image of figure 13a, in addition to movements of the left ventricle muscle (mainly upward) that enhances blood pumping, a thickening of myocardium muscle, especially in the lower

parts of the ventricle, is clearly seen. These effects are mainly due to stimulant electrical currents that are not modeled in this study. The agreement between the simulation results and the CT images in all views for time $\mathrm{t}=0.12 \mathrm{~s}$ is good and worsens at later time-steps. The overall agreement of the images is acceptable, and the main difference is the abnormal side contraction of the simulation images that might be caused by the absence of the vertical movement and thickening of the LV tissue, as mentioned above. Also, left ventricle systolic anterior motion is depicted in simulation results. Horizontal vectors represent this motion left and right in figure 13. This difference in ventricle shape after the start of systole perhaps shows the importance of electrical response modeling of the heart tissue, which is absent in the present study.

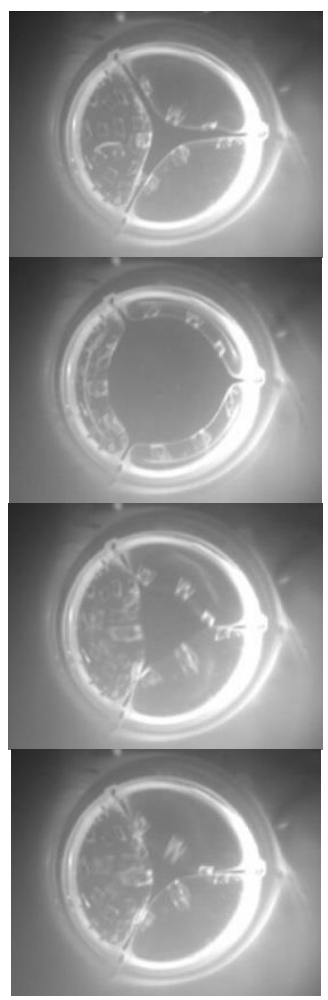

CT Scan images

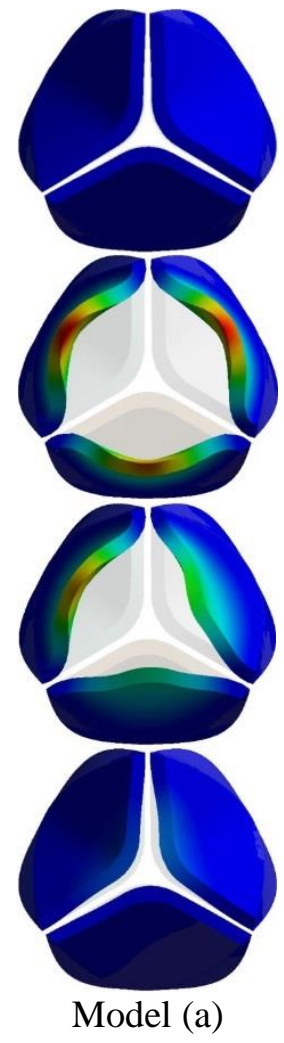

Model (a)

Figure 12 Comparison of deformation of the aortic valve for the performed simulation and CT scan images [24].

Volume 17, 2022 
(a)

CT Scan images of first ventricle

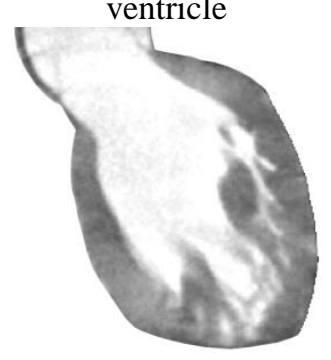

Time $=0.12 \mathrm{~s}$

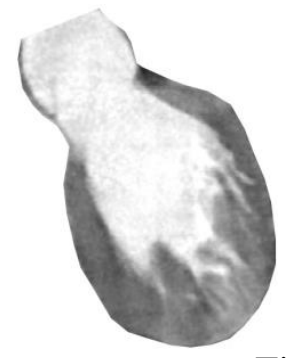

Time $=0.3 \mathrm{~s}$

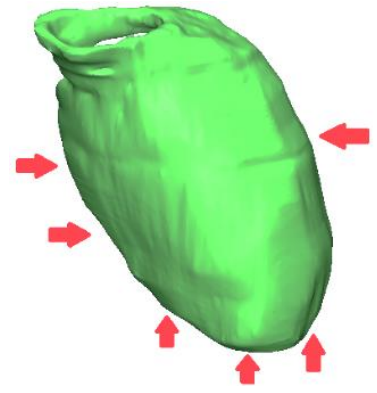

Time $=0.12 \mathrm{~s}$
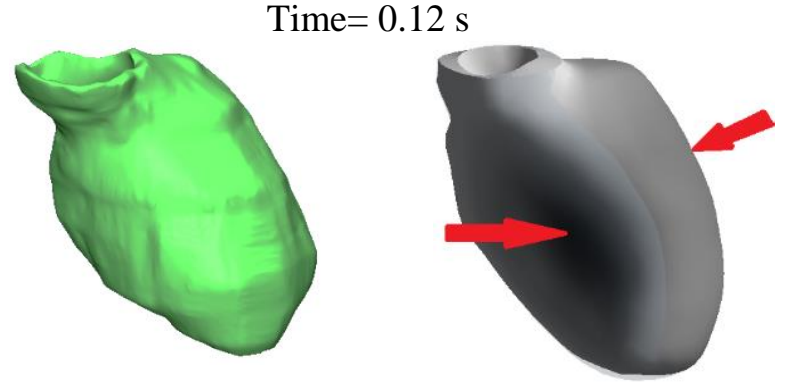

Time $=0.3 \mathrm{~s}$
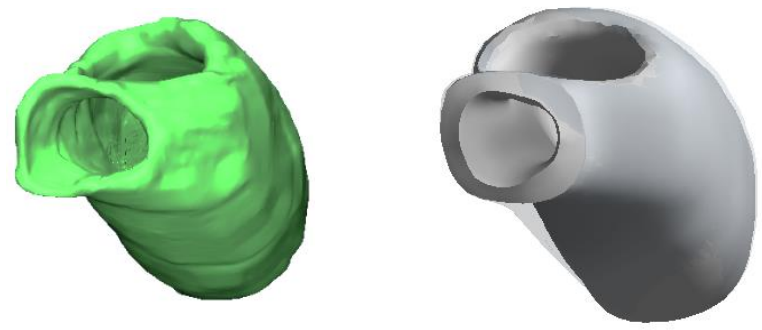

Time $=0.12 \mathrm{~s}$
Simulation of the first ventricle-pressure B.C
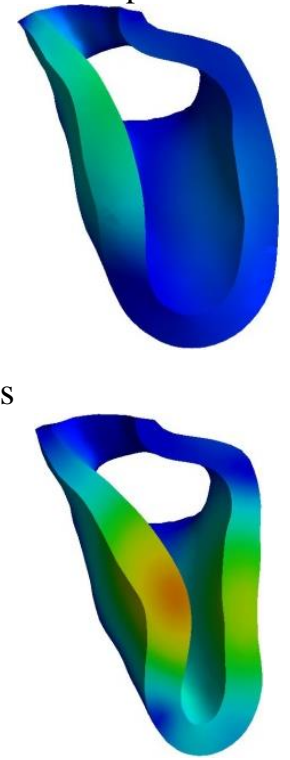

(b) Isometric view

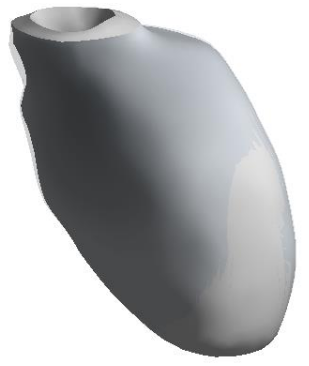

(c) Up view
CT Scan images of first ventricle

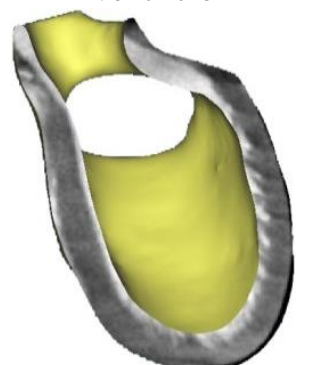

Time $=0 \mathrm{~s}$

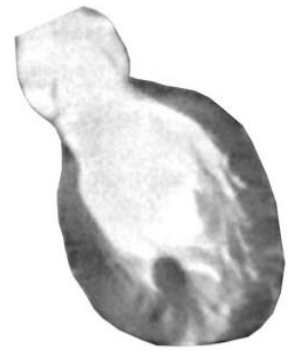

Time $=0.24 \mathrm{~s}$
Simulation of the first ventricle-pressure B.C
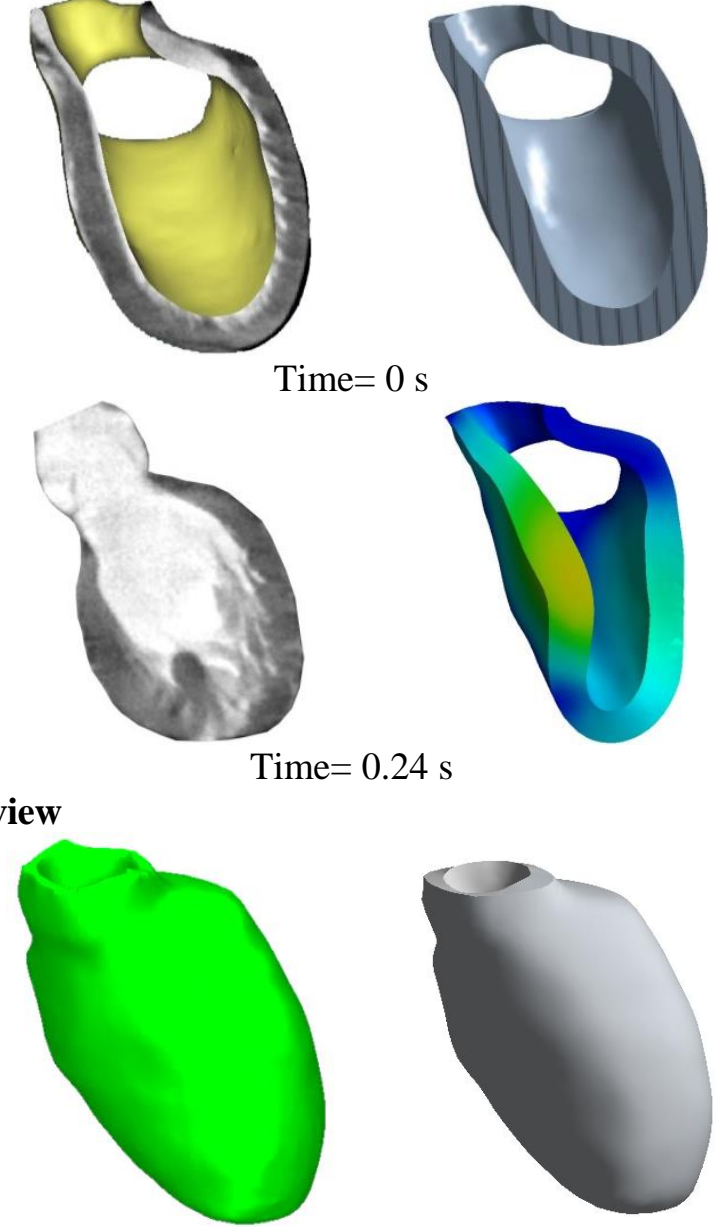

Time $=0 \mathrm{~s}$
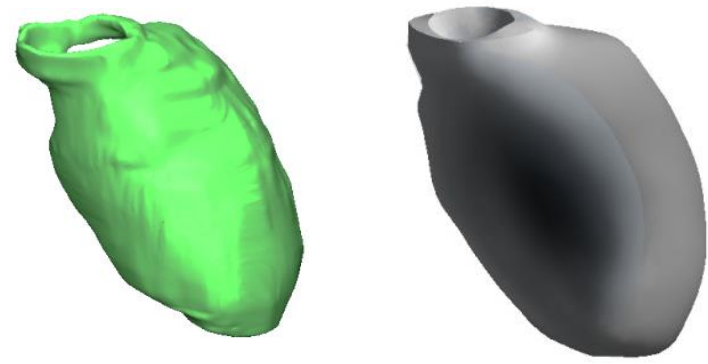

Time $=0.24 \mathrm{~s}$
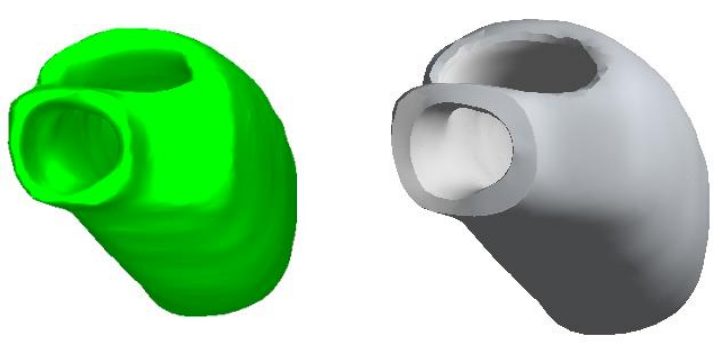

Time $=0 \mathrm{~s}$ 


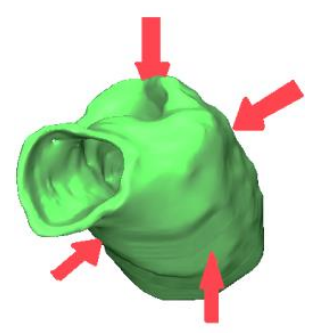

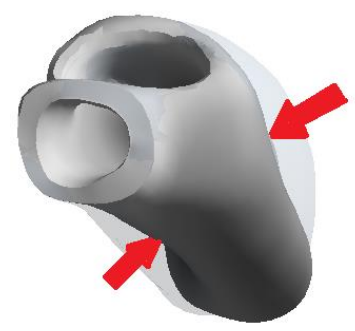

Time $=0.3 \mathrm{~s}$

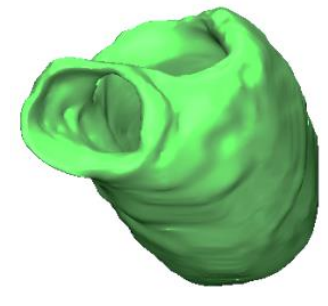

Time $=0.24 \mathrm{~s}$

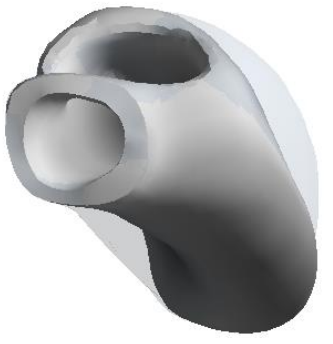

(d) Front view

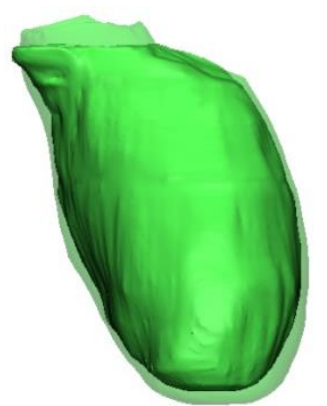

Time $=0.12 \mathrm{~s}$

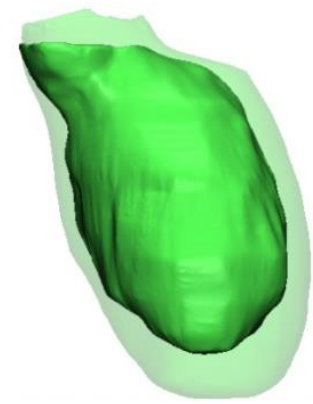

Time $=0.3 \mathrm{~s}$
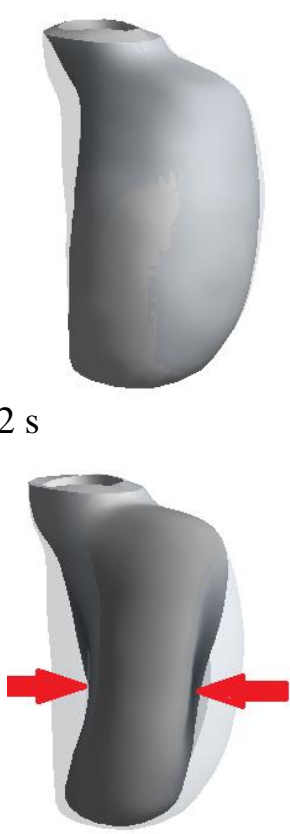

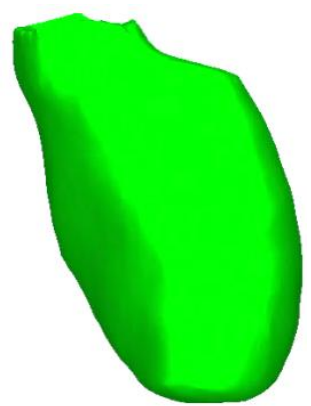

Time $=0 \mathrm{~s}$
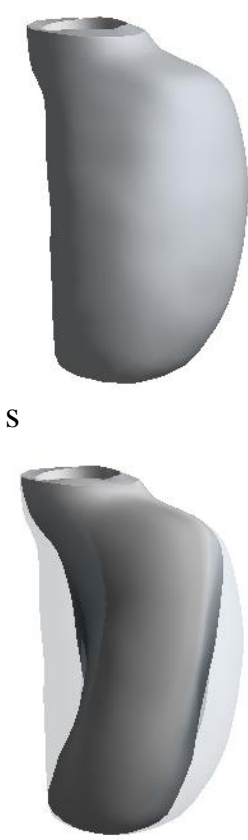

Time $=0.24 \mathrm{~s}$

Figure 13 Comparison of LV deformation based on simulation and CT scan images from different views.

\section{4-2 Fluid Domain}

Table 3 lists the computed hemodynamic parameters of $\mathrm{LV}$ along with the related

Table 3. Comparison of hemodynamic parameters of left ventricle with physiological values.

\begin{tabular}{|c|c|c|c|}
\cline { 2 - 4 } \multicolumn{1}{c|}{} & $\begin{array}{c}\text { Present study- } \\
\text { Pressure B.C- } \\
\text { First ventricle }\end{array}$ & $\begin{array}{c}\text { Present study- } \\
\text { velocity B.C- } \\
\text { First ventricle }\end{array}$ & Physiological range \\
\hline End-systolic volume (ml) & 76.485 & 73.102 & $58.1 \pm 30.1$ \\
\hline Stroke-volume (ml) & 62.166 & 65.92 & $55-100$ \\
\hline Cardiac output (l/min) & 4.74 & 5.017 & $4-8$ \\
\hline End-diastolic volume (ml) & 138.621 & 138.621 & $134.2 \pm 39.9$ \\
\hline Ejection fraction \% & 44.85 & 47.42 & $58.1 \pm 11.9$ \\
\hline
\end{tabular}

The maximum blood velocity at the outlet for a healthy aortic valve is smaller than 2 $\mathrm{m} / \mathrm{s}$, and the maximum cross-sectional area at the outlet of the aortic valve is $3.9 \pm 1.2$ physiological data [25-26]. All the computed parameters lie in the normal ranges. $\mathrm{cm}^{2}$ [27]. Regarding the velocity at plane 1 in figure 9a and the area ratio of the maximum opening of the aorta valve at $\mathrm{t}=0.12 \mathrm{~s}$ to the outlet area, the maximum 
velocity of $1.607 \mathrm{~m} / \mathrm{s}$ occurs at the aorta valve, which is in the normal physiological range. As shown in figure 14a, the mean velocity at the exit of the aortic valve is similar to the physiological reference chart [27]. The only difference is in the magnitude of maximum velocity. Although based on

- Velocity profile at the exit of the aortic valve [25]

- Velocity profile at the exit of the aortic valve - current simulation

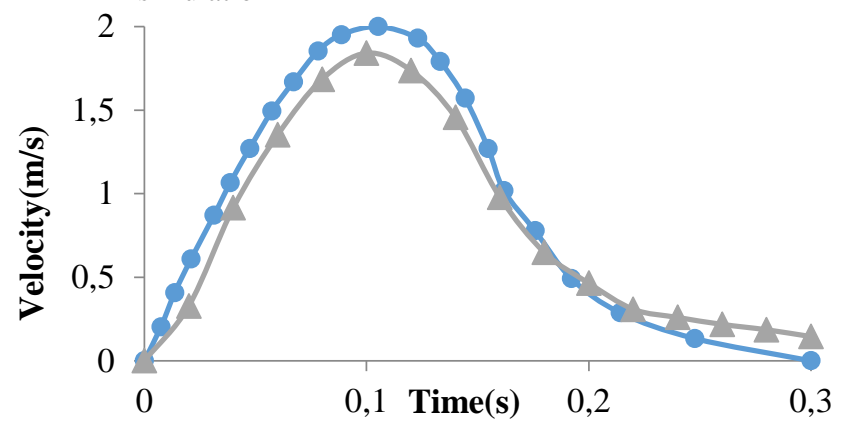

(a) the criteria that the maximum velocity should be less than $2 \mathrm{~m} / \mathrm{s}$ [27], the simulation results are acceptable. The maximum velocity at the output of the domain, which is the same as the ascending aorta, is presented in figure $14 \mathrm{~b}$.

- Velocity profile at the entrance of the aorta tube [25]
- The velocity profile at the entrance of the aortic tube current simulation

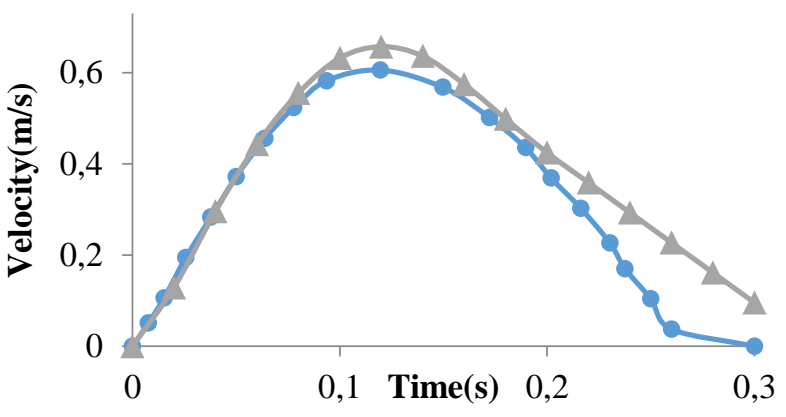

(b)

Figure 14 Velocity profiles (a) at the exit of the aortic valve (b) at the ascending aorta.

\section{4-3 HCM Results}

As mentioned earlier, under HCM condition the mitral valve leaflets approach towards the septum wall. As the leaflets move within a certain vicinity of the wall, some negative volume elements occur in the neighboring region. The HCM simulations are carried out for the beginning of the systole up until 0.3s to avoid this computational error. A significant parameter is named "coaptationto-septal distance" and is defined as the shortest distance between the coaptation point at the end-systole and the intraventricular septum. Figure 15 shows this distance and the geometry of the left ventricle with the two valves added.

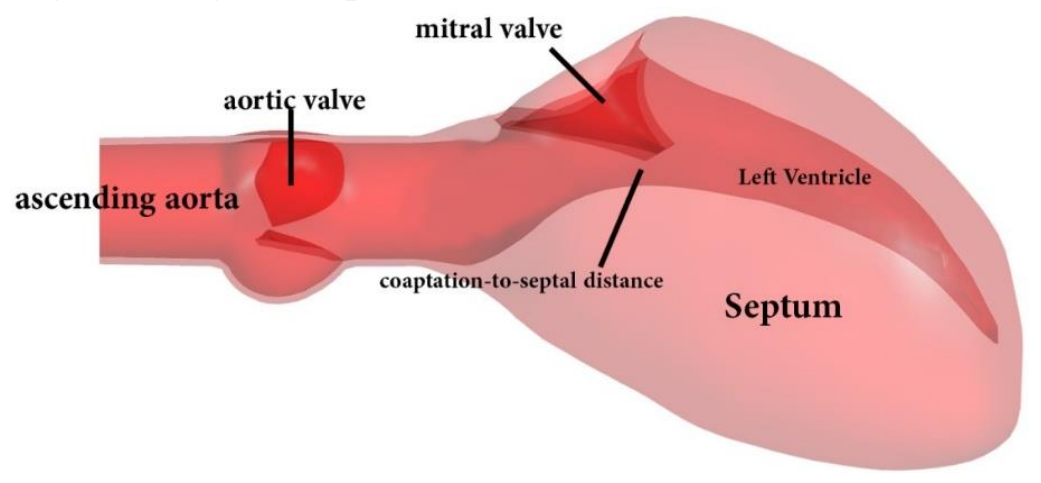

Figure 15 The coaptation-to-septal distance region and the geometry used for HCM simulation

Ventricular volume change is plotted for all the simulations in figure 16 to compare HCM results with the healthy condition. 


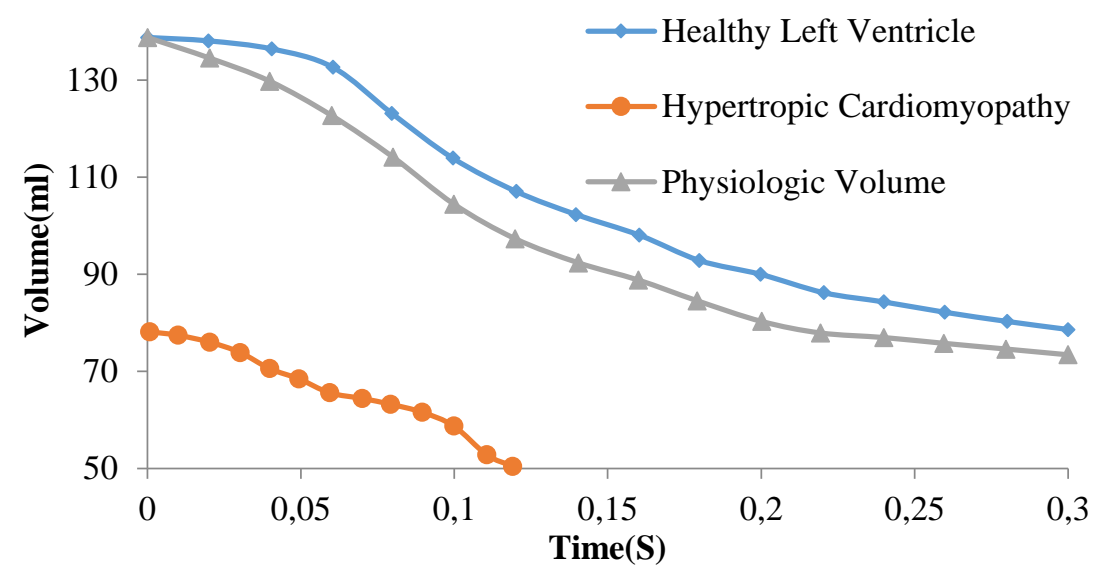

Figure 16 The left ventricular volume change for HCM condition.

Considering the whole cardiac cycle, the cardiac output for the HCM condition would be $2.123 \mathrm{lit} / \mathrm{min}$, which is much less than the
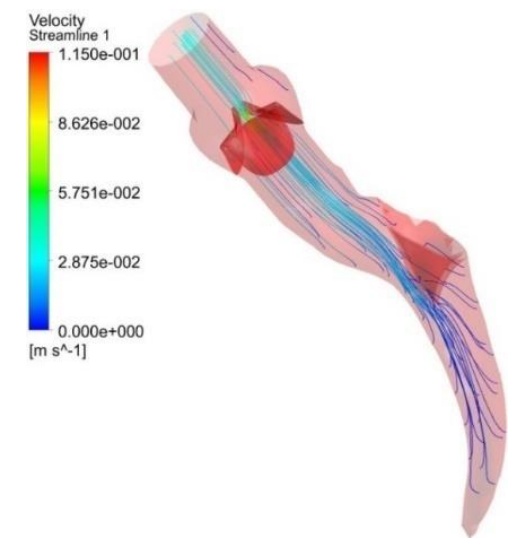

$\mathrm{t}=0.01 \mathrm{~s}$

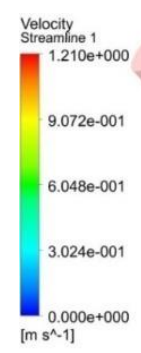

$\mathrm{t}=0.08 \mathrm{~s}$ physiological value under healthy condition, i.e., 4-8 lit/min. Figure 17 shows the 3D streamlines for laminar flow under HCM condition.
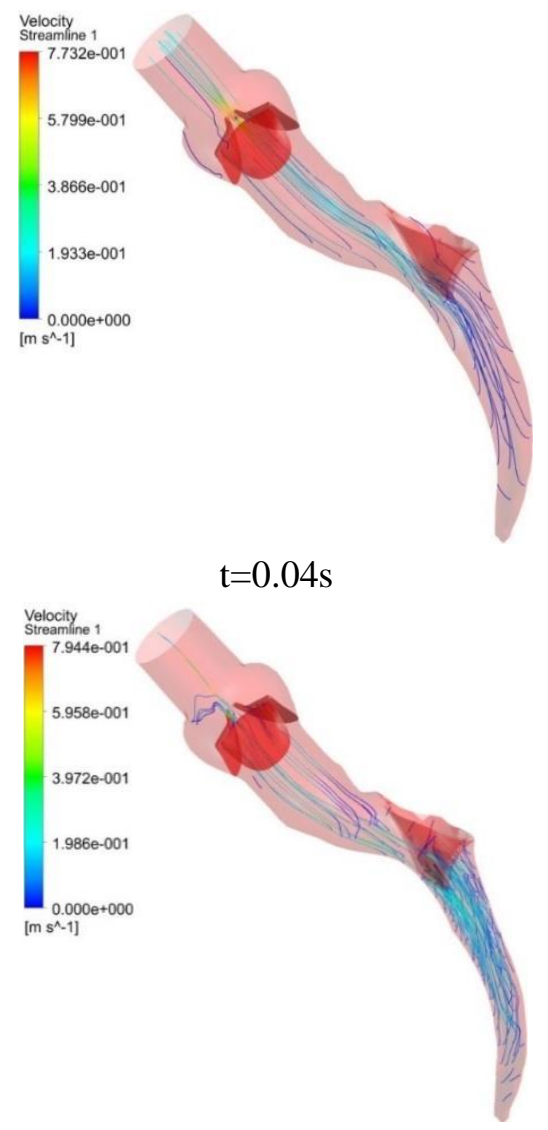

$\mathrm{t}=0.12 \mathrm{~s}$

Figure 17 3D streamlines for HCM condition

In order to study the interaction of the septum wall and mitral valve for the HCM case, figure 17 is presented. According to figure 17, the streamlines should rotate to pass the mitral valve and through the narrow 
region between the mitral valve leaflets and the septum wall (coaptation-to-septal distance). This kind of flow exerts a drag force on the mitral valve, which will make the anterior leaflet move towards the septum wall during the systolic anterior motion. Based on the figure 18 and compared with a healthy heart, the reduction in the crosssection area and the decrease of the local pressure along with the presence of the drag force on the posterior leaflet and the lower pressure on the anterior leaflet of a flexible valve bring about more blockage of the $\mathrm{LV}$ blood passage by the mitral valve. Consequently, the flexible mitral valve blockage results in stagnation pressure loss and weaker heart-pumping performance. Therefore, low local pressure and lower stagnation pressure after the mitral valve produce an adverse pressure gradient toward the aorta and may even cause reverse flow. This reverse flow is detected in simulation results at $\mathrm{t}=0.12 \mathrm{~s}$.

For HCM simulation, the maximum area of the aortic valve is $2.228 \mathrm{~cm}^{2}$ and occurs at $\mathrm{t}$ $=0.1 \mathrm{~s}$, while for healthy condition, the maximum area of the aortic valve occurs at the same time and is equal to $3.9 \pm 1.2 \mathrm{~cm}^{2}$. Moreover, the maximum velocity at the aorta inlet is $1.27 \mathrm{~m} / \mathrm{s}$ and $0.414 \mathrm{~m} / \mathrm{s}$ for healthy and HCM conditions, respectively. The comparison shows that under the HCM condition, the blood velocity at the aorta's inlet and the aortic valve's maximum openness lie outside the physiological ranges.

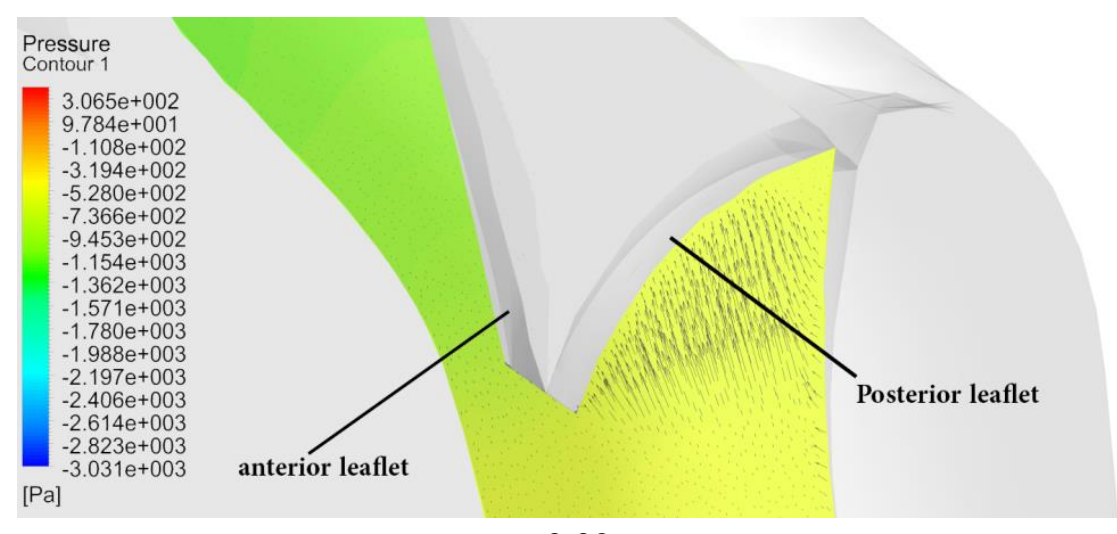

$$
\mathrm{t}=0.02 \mathrm{~s}
$$
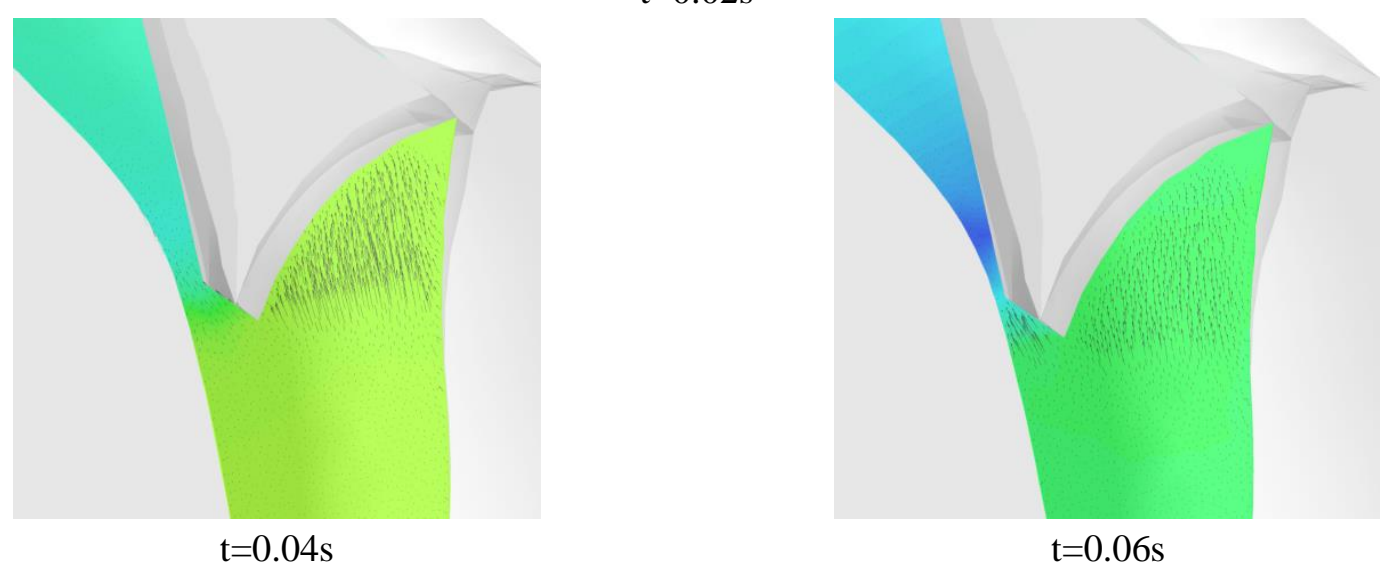

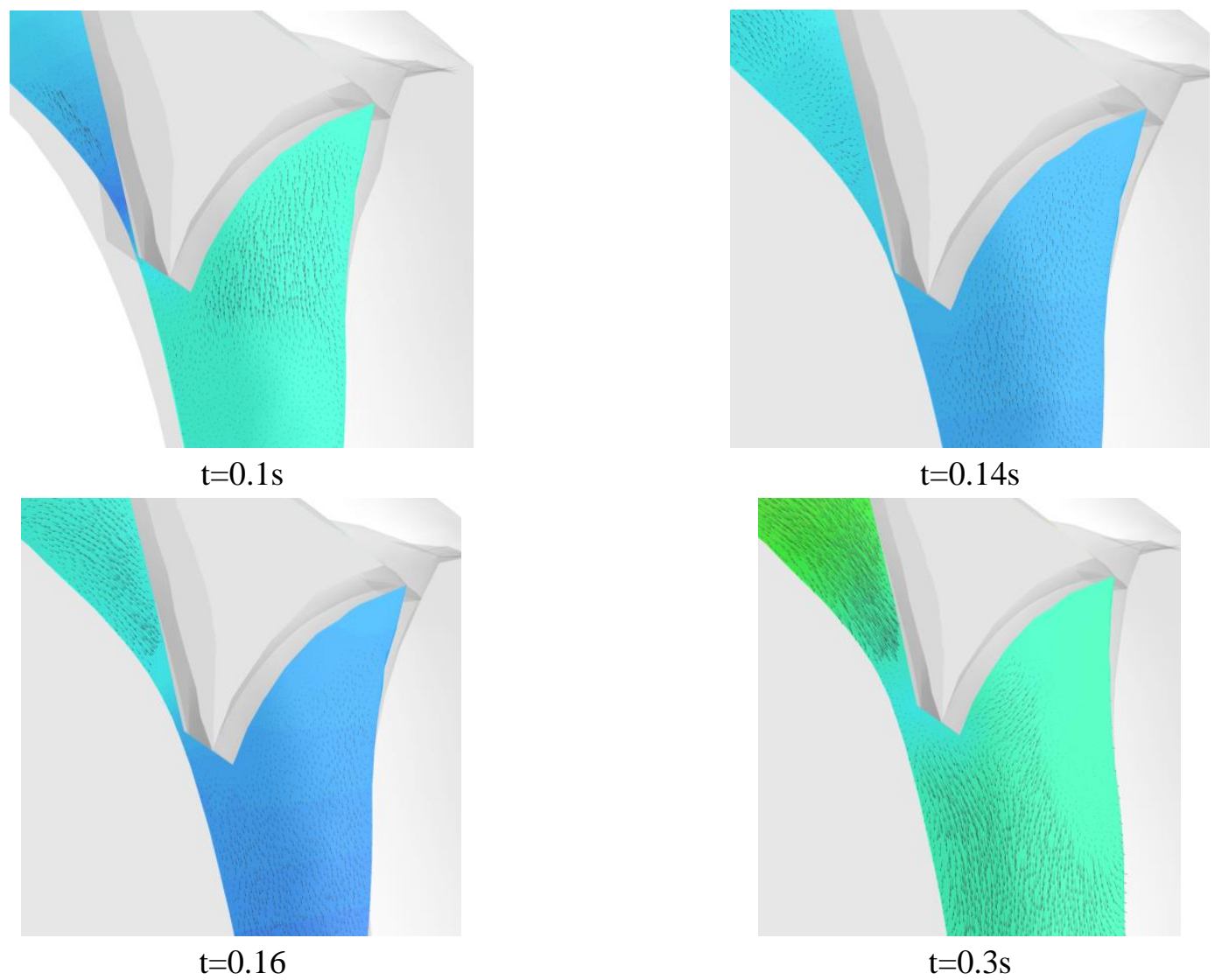

Figure 18 pressure contour around the coaptation-to-septal distance region for HCM condition

As shown in figure 18, the blood flow initially moves to the aortic valve. It is observed that during systole, the blood flow behind the posterior leaflet of the mitral valve moves it to the left ventricular wall (septum). Streamline vectors in this form are visible behind the posterior leaflet of the mitral valve. The drag force on the mitral valve causes it to move towards the septum and reduces the distance between the ventricular wall and the anterior leaflet of the mitral valve. This distance is called coaptation-to-septal distance. Another prominent factor seen in the images of figure 18 is the pressure gradient around the mitral valve. Before the 0.12 second, as the leaflets approach the septum, the pressure after the mitral valve decreases. This reduction in pressure after the mitral valve is another factor that causes the anterior leaflet of the mitral valve to move toward the septum. The coaptation-to-septal distance is reduced to the point that after the time $0.12 \mathrm{~s}$ and the creation of stagnation pressure, the flow begins to rotate after the mitral valve, and the pressure gradient is reversed. At this time, a reverse flow occurs.

\section{Conclusion}

The precise and correct simulation of the human heart can be beneficial to estimate the heart performance and cardiac output under different in vivo conditions. In this study, real CT scan images are used to construct an actual shaped model of human LV under the healthy and HCM disease conditions. Using the FSI method, the blood flow in LV and ventricular tissue and aortic valve deformations are simulated during the 
systole period of the cardiac cycle for two models; the healthy LV and HCM model.

The 3D grid is built for physical models, and the discretized equations are solved for both solid and fluid domains. The grid independence analysis is performed for both models. Also, the time independence of numerical outcomes is investigated to ensure obtained results' accuracy. Two simulations with different BCs at the aortic outlet are carried out for the healthy model. The velocity and pressure outlet BCs are defined based on the physiological charts. The results for left ventricular volume change for these simulations are approximately the same and in accordance with available physiological data of LV volume change during systole. While these two models produce similar approximate flow and solid results for systole, another study for the diastole phase of the $\mathrm{LV}$ showed different results for the velocity (imprecise) and pressure BC (precise) models. The explanation would be the strong effect of the heart electrical current on the deformations, which is presented during systole and not modeled in the present study, and the absence of this effect in the diastole phase.

For the healthy model, numerical results capture the systolic anterior motion of $\mathrm{LV}$ identical to the CT scan images. The blood streamlines in LV display the local pressure variation near the aortic valve leaflets during the anterior motion. The results for the solid domain show the good accordance between FSI results for LV shape deformation and CT scan images. Besides, the FSI results for the aortic valve leaflets deformation are analogous with available CT scan images of this valve during systole. The maximum deformation of the aortic valve occurred at the time of $0.12 \mathrm{~s}$; simultaneously, the output velocity is maximum, and the aortic leaflet experiences the maximal opening. The maximum opening surface of the aortic valve is $4.38 \mathrm{~cm} 2$ which is consistent with physiological observation on a healthy individual.

For the HCM model, the maximum opening of the aortic valve is $2.228 \mathrm{~cm}^{2}$, which is not in the physiological range, indicating the drastic effect of HCM on the performance of the aortic valve. Moreover, the ventricular volume change decreases significantly from healthy to HCM condition. Compatible with the pathological nature of the HCM, under the severe malady, the LV loses its pumping ability to send blood into the aorta. Accordingly, the maximum blood velocity at the aortic outlet for HCM condition is 0.414 $\mathrm{m} / \mathrm{s}$ while this velocity is $1.27 \mathrm{~m} / \mathrm{s}$ for the healthy condition. The comparison indicates that under the HCM disorder, the blood velocity at the aorta's inlet and the aortic valve's maximum openness lie outside the physiological spans.

In conclusion, the FSI simulation can undoubtedly lead to feasible results for $\mathrm{LV}$ simulation when relevant physical and numerical details are considered. More complete heart models considering heart electrical current and subsequent tissue deformation would eventually provide the necessary numerical tool for practical applications. 


\section{References}

[1] M. Alishahi, M. Alishahi and H. Emdad, "Numerical simulation of blood flow in a flexible stenosed abdominal real aorta," Scientia Iranica, vol. 18, no. 6, pp. 1297-1305, 2011.

[2] S. Numata, K. Itatani, K. Kanda, K. Doi, S. Yamazaki, K. Morimoto, K. Manabe, K. Ikemoto and H. Yaku, "Blood flow analysis of the aortic arch using computational fluid dynamics," European Journal of CardioThoracic Surgery, vol. 49, no. 6, pp. 15781585, 2016.

[3] F. Ismail, M. Shukri Zakaria, M. Tamagawa, A. Fazli Abdul Azi, S. Wiriadidjaya, A. Azrif Basri and K. Arifin Ahmad, "Computational fluid dynamics study of blood flow in aorta using OpenFOAM," Journal of Advanced Research in Fluid Mechanics and Thermal Sciences, vol. 34, no. 1, pp. 81-89, 2018.

[4] T. B. Le and F. Sotiropoulos, "Fluid-structure interaction of an aortic heart valve prosthesis driven by an animated anatomic left ventricle," Journal of computational physics, vol. 244, pp. 41-62, 2013.

[5] H. Watanabe, S. Sugiura, H. Kafuku and T. Hisada, "Multiphysics simulation of left ventricular filling dynamics using fluid-structure interaction finite element method," Biophysical journal, vol. 87, no. 3, pp. 2074-2085, 2004.

[6] M.-H. Moosavi, N. Fatouraee, H. Katoozian, A. Pashaei, O. Camara and A. F. Frangi, "Numerical simulation of blood flow in the left ventricle and aortic sinus using magnetic resonance imaging and computational fluid dynamics," Computer methods in biomechanics and biomedical engineering, vol. 17, no. 7, pp. 740-749, 2014.

[7] C. Lorenz and J. von Berg, "A comprehensive shape model of the heart," Medical image analysis, vol. 10, no. 4, pp. 657-670, 2006.

[8] H. S. Kim, "Nonlinear multi-scale anisotropic material and structural models for prosthetic and native aortic heart valves," Georgia Institute of Technology, Georgia, 2009.
[9] M. S. Sacks, W. D. Merryman and D. E. Schmidt, "On the biomechanics of heart valve function," Journal of biomechanics, vol. 42, no. 12, pp. 1804-1824, 2009.

[10] T. B. Le and F. Sotiropoulos, "Fluid-structure interaction of an aortic heart valve prosthesis driven by an animated anatomic left ventricle," Journal of computational physics, vol. 244, pp. 41-62, 2013.

[11] F. Sturla, E. Votta, M. Stevanella, C. A. Conti and A. Redaelli, "Impact of modeling fluidstructure interaction in the computational analysis of aortic root biomechanics," Medical engineering \& physics, vol. 35, no. 12, pp. 17211730, 2013.

[12] M.-C. Hsu, D. Kamensky, Y. Bazilevs, M. S. Sacks and T. J. Hughes, "Fluid-structure interaction analysis of bioprosthetic heart valves: significance of arterial wall deformation," Computational mechanics, vol. 54, no. 4, pp. 1055-1071, 2014.

[13] E. Braunwald, C. E. Seidman and U. Sigwart, "Contemporary evaluation and management of hypertrophic cardiomyopathy," Circulation, vol. 106, no. 11, pp. 1312-1316, 2002.

[14] A. Moghaddaszade-Kermani, P. Oshkai and A. Suleman, "Fluid-Structure Interaction Simulation of Blood Flow Inside a Diseased Left Ventricle With Obstructive Hypertrophic Cardiomyopathy in Early Systole," In Fluids Engineering Division Summer Meeting, vol. 43727, pp. 1799-1807, 2009.

[15] W. Sun, K. Li and E. Sirois, "Simulated elliptical bioprosthetic valve deformation: implications for asymmetric transcatheter valve deployment," Journal of biomechanics, vol. 43, no. 16, pp. 3085-3090, 2010.

[16] J. V. Soulis, G. D. Giannoglou, Y. S. Chatzizisis, K. V. Seralidou, G. E. Parcharidis and G. E. Louridas, "Non-Newtonian models for molecular viscosity and wall shear stress in a 3D reconstructed human left coronary artery," Medical engineering \& physics 30, no. 1 (): ., vol. 30, no. 1, pp. 9-19, 2008.

[17] Y.-c. Fung, Biomechanics: circulation, Springer Science \& Business Media, 2013. 
[18] V. Prot, B. Skallerud, G. Sommer and G. A. Holzapfel, "On modelling and analysis of healthy and pathological human mitral valves: two case studies," Journal of the mechanical behavior of biomedical materials, vol. 3, no. 2, pp. 167-177, 2010.

[19] C. Yang, D. Tang, I. Haber, T. Geva and J. Pedro, "In vivo MRI-based 3D FSI RV/LV models for human right ventricle and patch design for potential computer-aided surgery optimization," Computers \& structures, vol. 85, no. 11, pp. 988-997, 2007.

[20] A. M. Pouch, C. Xu, P. A. Yushkevich, A. S. Jassar, M. Vergnat, J. H. Gorman, R. C. Gorman, C. M. Sehgal and B. M. Jackson, "Semi-automated mitral valve morphometry and computational stress analysis 3D ultrasound," Journal of biomechanics, vol. 45, no. 5, pp. 903-907, 2012.

[21] M. S. Arefin and Y. S. Morsi, "Fluid structure interaction (FSI) simulation of the left ventricle (LV) during the early filling wave (E-wave), diastasis and atrial contraction wave (A-wave)," Australasian physical \& engineering sciences in medicine, vol. 37, no. 2, pp. 413-423, 2014.

[22] K. Sagawa, "The ventricular pressure-volume diagram revisited," Circulation Research, vol. 43, no. 5, pp. 677-687, 1978.

[23] S. W. Fielden, B. K. Fornwalt, M. Jerosch- Herold, R. L. Eisner, A. E. Stillman and J. N. Oshinski, "A new method for the determination of aortic pulse wave velocity using cross- correlation on 2D PCMR velocity data," Journal of Magnetic Resonance Imaging, vol. 27, no. 6, pp. 1382-1387, 2008.

[24] R. Haj-Ali, L. P. Dasi, H.-S. Kim, J. Choi, H. W. Leo and A. P. Yoganathan, "Structural simulations of prosthetic tri-leaflet aortic heart valves," Journal of biomechanics, vol. 41, no. 7, pp. 1510-1519, 2008.

[25] T. Schlosser, K. Pagonidis, C. U. Herborn, P. Hunold, K.-U. Waltering, T. C. Lauenstein and J. Barkhausen, "Assessment of left ventricular parameters using 16-MDCT and new software for endocardial and epicardial border delineation," American Journal of Roentgenology, vol. 184, no. 3, pp. 765-773, 2005.
[26] P. B. Raven, D. H. Wasserman, W. G. Squires and T. D. Murray, Exercise Physiology, Nelson Education, 2012.

[27] R. Halevi, A. Hamdan, G. Marom, K. Lavon, S. Ben-Zekry, E. Raanani, D. Bluestein and R. HajAli, "Fluid-structure interaction modeling of calcific aortic valve disease using patientspecific three-dimensional calcification scans," Medical \& biological engineering \& computing, vol. 54, no. 11, pp. 1683-1694, 2016.

[28] D. Chen, M. Müller-Eschner, H. von TenggKobligk, D. Barber, D. Böckler, R. Hose and Y. Ventikos, "A patient-specific study of type-B aortic dissection: evaluation of true-false lumen blood exchange," Biomedical engineering online, vol. 12, no. 1, pp. 1-16, 2013.

\section{Contribution of individual authors to the creation of a scientific article}

Mohammad Monfared carried out the simulation and writing down the manuscript. Prof. Mohammad Mehdi Alishahi was the academic supervisor who guided the research.

Marzieh Alishahi wrote the final manuscript and was responsible with editing process.

\section{Sources of funding for research presented in a scientific article or scientific article itself}

There is no specific source of funding for this research. 